Cochrane Database of Systematic Reviews

\title{
Brexpiprazole versus placebo or other antidepressive agents for treating depression (Protocol)
}

Ralovska S, Koychev I, De Crescenzo F, Marinov P, Cipriani A

Ralovska S, Koychev I, De Crescenzo F, Marinov P, Cipriani A.

Brexpiprazole versus placebo or other antidepressive agents for treating depression (Protocol).

Cochrane Database of Systematic Reviews 2021, Issue 2. Art. No.: CD013866.

DOI: 10.1002/14651858.CD013866.

www.cochranelibrary.com 
TABLE OF CONTENTS

HEADER 1

ABSTRACT

BACKGROUND

OBJECTIVES

METHODS

ACKNOWLEDGEMENTS

REFERENCES

HISTORY

CONTRIBUTIONS OF AUTHORS

DECLARATIONS OF INTEREST

SOURCES OF SUPPORT 
[Intervention Protocol]

\section{Brexpiprazole versus placebo or other antidepressive agents for treating depression}

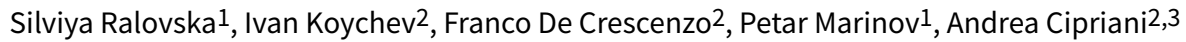

1Department of Neurology, Psychiatry, Physiotherapy and Rehabilitation, Preventive Medicine, and Public Health, Sofia University "St. Kliment Ohridski”, Sofia, Bulgaria. 2Department of Psychiatry, University of Oxford, Oxford, UK. ${ }^{3}$ Oxford Health NHS Foundation Trust, Warneford Hospital, Oxford, UK

Contact address: Andrea Cipriani, andrea.cipriani@psych.ox.ac.uk.

Editorial group: Cochrane Common Mental Disorders Group.

Publication status and date: New, published in Issue 2, 2021.

Citation: Ralovska S, Koychev I, De Crescenzo F, Marinov P, Cipriani A. Brexpiprazole versus placebo or other antidepressive agents for treating depression (Protocol). Cochrane Database of Systematic Reviews 2021, Issue 2. Art. No.: CD013866. DOI: 10.1002/14651858.CD013866.

Copyright @ 2021 The Cochrane Collaboration. Published by John Wiley \& Sons, Ltd.

\section{A B S T R A C T}

\section{Objectives}

This is a protocol for a Cochrane Review (intervention). The objectives are as follows:

To investigate the efficacy, tolerability, acceptability, and safety of brexpiprazole as monotherapy or adjunct treatment in the acute- and long-term treatment of major depression. 


\section{B A C K G R O U N D}

\section{Description of the condition}

Major depressive disorder (MDD) is a chronic mental health condition that affects over 163 million people worldwide; it is the third cause of disability in the global burden of disease (GBD 2017). Persistent low mood and diminished interest or pleasure in daily activities form the core symptoms of the disorder; they are accompanied by significant weight loss, fatigue or loss of energy, psychomotor retardation (slowing of mental and physical activity), feelings of worthlessness, excessive or inappropriate guilt, diminished ability to concentrate, and suicidal ideation (APA 2013). According to the most recent edition of the Diagnostic and Statistical Manual of Mental Disorders (DSM V), five or more symptoms, including at least one of the main symptoms, and an episode duration of two or more weeks, are required for the diagnosis of MDD (APA 2013).

The treatment of depression includes pharmacological and psychological interventions, electroconvulsive therapy, and other treatment options. The choice of treatment for each person depends on various factors, such as the severity and duration of the depressive episode, response to treatment, risk-benefit ratio of the intervention, the person's preferences (NICE 2009). According to international guidelines, antidepressants are often prescribed as first-line treatment for moderate to severe episodes of major depression (APA 2010; Kennedy 2016; NICE 2009). Based on slight differences in their pharmacological properties, antidepressants are grouped into classes, such as selective serotonin reuptake inhibitors, selective serotonin and noradrenaline reuptake inhibitors, tricyclic antidepressants, and monoamine-oxidase inhibitors; (APA 2010)). On average, antidepressants are more efficacious than placebo for the acute treatment of depression in adults (Cipriani 2018). However, people with MDD vary substantially in their response rates (Kennedy 2016); when treated with a single antidepressant, as many as $50 \%$ of people with MDD do not reach a response threshold (Salanti 2016), measured as a $50 \%$ reduction of the score on a validated scale for depression (Bauer 2002). A variety of terms have been used to describe the degree of non-responsiveness to treatment, ranging from non-response to partial response (Bauer 2002; Papakostas 2020). A person's depression is generally considered to be treatment-resistant when it does not respond to a course of two or more antidepressants of adequate dose and duration (Malhi 2016), although more specific criteria, such as additional lack of response to psychotherapy, have also been suggested (Fava 2020). Currently, in cases in which MDD fails to respond to antidepressant monotherapy, switching to another antidepressant, adjunctive treatment (i.e. added treatment to assist the primary treatment), psychotherapy, or neuro stimulation, have been recommended (Kennedy 2016; NICE 2009)

\section{Description of the intervention}

In July 2015, the US Food and Drug Administration (FDA) approved brexpiprazole as a monotherapy for the treatment of schizophrenia, and as adjunct therapy for treatment-resistant depression in the USA (FDA 2015). In Europe, it is indicated only for the treatment of schizophrenia (EMA 2018). Brexpiprazole is a second-generation antipsychotic agent. It is a partial agonist of serotonin $5 \mathrm{HT}-1 \mathrm{~A}$ and dopamine $\mathrm{D} 2$ receptors, and an antagonist of serotonin $5 \mathrm{HT}-2 \mathrm{~A}$ receptors. It also has an antagonistic action with moderate affinity for the adrenergic $a 1 / 2-R$, histamine $H 1$ receptors, and a low affinity for the muscarinic $M$ receptors (Maeda 2014a; Otsuka 2020). Peak plasma concentration of brexpiprazole is achieved four hours after dose administration, with 95\% bioavailability. Its terminal half-life is 91 hours; the recommended regimen is once daily, $2 \mathrm{mg}$ to $4 \mathrm{mg} /$ day for schizophrenia, and $0.5 \mathrm{mg}$ to $3 \mathrm{mg} /$ day as add-on treatment for depression (Otsuka 2020). Brexpiprazole is metabolised primarily by CYP2D6 and CYP3A4 enzymes, and therefore, requires dosage adjustment when administered in people with poor CYP2D6 metabolism, or concomitantly with medications which inhibit CYP2D6, as well as with medications that induce or inhibit CYP3A4. (Otsuka 2020).

\section{How the intervention might work}

The mechanism by which brexpiprazole produces its antidepressive effects remains unclear. Preclinical studies compared brexpiprazole to aripiprazole (Maeda 2014a; Maeda 2014b; Stahl 2016), which has a similar pharmacological profile and has also been approved as augmentation treatment for TRD (FDA 2007).

Compared to aripiprazole, brexpiprazole has a lower intrinsic affinity for D2-receptors, and a stronger affinity for serotonin $5 \mathrm{HT}-1 \mathrm{~A}, 5 \mathrm{HT}-2 \mathrm{~A}$, and adrenergic a1B receptors, which is associated with a lower incidence of activation and extrapyramidal symptoms (Stahl 2016). Brexpiprazole has also a lower affinity for histamine $\mathrm{H} 1$ receptors, which could lead to lower rates of sedation, and weight gain (Maeda 2014a; Stahl 2016). On the basis of these specific actions on dopamine D2, serotonin 5HT-1A, 5HT-2A, adrenergic $\alpha 1 B$, and histamine $H 1$ receptors, it was suggested that brexpiprazole should be equally efficacious to aripiprazole as adjunctive treatment for depression, while causing fewer side effects (Stahl 2016).

A preclinical study of cognition in animal models of schizophrenia, sponsored by the manufacturer, found that brexpiprazole led to better cognitive performance in executive function domains than aripiprazole, and suggested this was due to its unique affinities for dopamine D2, serotonin $5 \mathrm{HT}-2 \mathrm{~A}$, and $5 \mathrm{HT}-1 \mathrm{~A}$ receptors (Maeda 2014b). Brexpiprazole as adjunct treatment led to improved cognition and functioning in a recent open-label clinical study on major depression (Fava 2017).

To investigate the efficacy and safety of brexpiprazole as addon treatment for MDD in clinical settings, a short-term Phase III, double-blind randomised controlled trial (RCT), sponsored by Otsuka Pharmaceutical Co., randomised 379 people with major depression who did not respond to an eight-week course of antidepressant monotherapy, to brexpiprazole or placebo as add-on treatment for six weeks (NCT01360645). Brexpiprazole 2 mg improved depression symptoms on the Montgomery-Åsberg Depression Rating Scale (MADRS) more than placebo (mean difference (MD) -3.12, 95\% confidence interval (Cl) -4.70 to -1.54 ; $P<0.0001)$. Those who received brexpiprazole more often had weight gain (7.98\% (brexpiprazole group) versus 3.14\% (placebo group)), and more often had akathisia, a 'subjective feeling of motor restlessness manifested by a compelling need to be in constant movement' (Sadock 2015) (7.45\% (brexpiprazole group) versus $1.05 \%$ (placebo group)).

Another short-term Phase III RCT, with a similar design, suggested that the efficacy and tolerability of brexpiprazole in people with 
depression might be dose-dependent (NCT01360632). In that study, 1539 people were randomised to brexpiprazole $1 \mathrm{mg}$, brexpiprazole $3 \mathrm{mg}$, or placebo, adjunctive to an antidepressant. Brexpiprazole $1 \mathrm{mg}$ failed to reduce depression symptoms on the MADRS compared to placebo (MD $-1.30,95 \% \mathrm{Cl}-2.73$ to 0.13 ; P $<0.0737$ ). Brexpiprazole $3 \mathrm{mg}$ was more efficacious than placebo on the MADRS (MD -1.95, 95\% Cl -3.39 to -0.51 ; $\mathrm{P}<0.0079$ ), but participants reported higher rates of akathisia (13.5\%) than those who received $1 \mathrm{mg}(4.4 \%)$. These results suggested that higher dosages of brexpiprazole might be more efficacious, but less tolerable than lower dosages.

\section{Why it is important to do this review}

In cases of an inadequate response to an antidepressant, recommendations for adjunct medications include an antipsychotic, another antidepressant, lithium, triiodothyronine, stimulants (Kennedy 2016). Brexpiprazole is the newest antipsychotic to receive approval as an adjunct treatment for MDD, with several reviews of its efficacy and safety in depression already published (Citrome 2015; Kishi 2019; Mclntyre 2016; Nelson 2018; Thase 2016; Thase 2019; Yoon 2017). While informative, these reviews have a number of limitations.

First, all existing reviews, except Kishi 2019, explored only shortterm effects (up to six-week follow-up), and were limited by the small and selective number of RCTs included. For instance, only two RCTs were included in some reviews (Citrome 2015; McIntyre 2016; Thase 2016; Yoon 2017); Thase 2019 included four. Second, none of these reviews assessed the effect of brexpiprazole on depression in people under the age of 18 years. Third, some of these reviews mentioned a review protocol, but it was often not available, or was not easy to access. Fourth, several reviews were sponsored by the manufacturer of brexpiprazole (McIntyre 2016; Nelson 2018; Thase 2016; Thase 2019). What is more, these reviews lacked a formal assessment of risk of bias. Finally, out of all of these reviews, only one evaluated the quality of the evidence with GRADE methodology (Yoon 2017).

As there are more studies investigating the effect of brexpiprazole, we need a high quality review to properly assess the efficacy, tolerability, acceptability, and safety of brexpiprazole as treatment for depression, and to guide clinical practice.

\section{OB JECTIVES}

To investigate the efficacy, tolerability, acceptability, and safety of brexpiprazole as monotherapy or adjunct treatment in the acuteand long-term treatment of major depression.

\section{METHODS}

\section{Criteria for considering studies for this review}

\section{Types of studies}

We will include only double-blind, randomised controlled trials (RCTs), cross-over RCTs, and cluster-RCTs. We will include both published and unpublished studies,

We will exclude quasi-randomised controlled trials, in which allocation is decided by an approximation of randomisation (i.e. by alternate days, medical record number).

\section{Types of participants \\ Diagnosis}

Participants with a primary diagnosis of major depressive disorder (MDD), according to DSM-IV (APA 1994), DSM-IV-TR (APA 2000), DSM-5 (APA 2013), ICD-10 (WHO 1992), Feighner criteria (Feighner 1972), Research Diagnostic Criteria (Spitzer 1978), or any other standardised criteria for MDD.

We decided not to consider ICD-9 (WHO 1978) for the diagnosis, because it lacked operationalised criteria. We will exclude studies including more than $20 \%$ of participants with diagnosis of bipolar depression or psychotic depression (Furukawa 2016).

\section{Characteristics}

We will include trials with participants of any age, of both sexes, and any ethnicity.

We will include trials with people with MDD who did not respond to previous antidepressant treatment, and who are still experiencing acute depressive symptoms. For the purpose of the analysis, we will divide participants into the following groups:

- participants with history of an inadequate response (i.e. participants who reported a less than $50 \%$ reduction of the score on a standardised depression scale after treatment with one antidepressant as monotherapy, at adequate dose and duration (Papakostas 2020)

- participants with treatment-resistant depression (i.e. participants who did not respond to two or more trials with antidepressant monotherapy, at adequate dose and duration (Malhi 2016)

\section{Comorbidities}

We will exclude RCTs in which any participants have a concurrent primary diagnosis of another mental disorder, but we will not exclude trials in which participants have a secondary diagnosis of a mental disorder (Furukawa 2016). We will exclude RCTs including participants with serious medical illness (such as myocardial infarction, uncontrolled diabetes mellitus).

\section{Setting}

Any setting (i.e. inpatients, general practice and outpatient specialist services, residents in care homes or nursing centres).

\section{Types of interventions}

\section{Experimental intervention}

Brexpiprazole (either as monotherapy or add-on therapy). We will include studies on brexpiprazole in the licensed dose range for the treatment of depression $(0.5 \mathrm{mg}$ to $3 \mathrm{mg}$ ). We will also include studies with higher doses, as higher doses of the medication (4 mg) were approved for another indication (FDA 2015), and plasma concentrations may vary depending on differences in drug metabolism and co-medication (Otsuka 2020). We will include treatment arms with fixed and flexible dosage regimens.

\section{Comparator interventions}

i. Antidepressants (as monotherapy or add-on therapy), including, but not limited to: 
- Selective serotonin reuptake inhibitors (SSRIs) - sertraline, paroxetine, citalopram, escitalopram, fluoxetine, fluvoxamine

- Serotonin-norepinephrine reuptake inhibitors (SNRIs) - venlafaxine, duloxetine, desvenlafaxine, milnacipran, levomilnacipran

- Tricyclic antidepressants (TCAs) - including amitriptyline, amoxapine, clomipramine, desipramine, dosulepin or dothiepin, doxepin, imipramine, lofepramine, maprotiline, nortriptyline, protriptyline, trimipramine

- Monoamine oxidase inhibitors (MAOIs) - phenelzine, isocarboxazide, tranylcypromine, moclobemide, brofaromine

- Other antidepressants - mirtazapine, agomelatine, trazodone, vortioxetine, bupropion, reboxetine, esketamine, vilazodone

ii. Placebo (as monotherapy or add-on therapy)

\section{Types of outcome measures}

\section{Primary outcomes}

1. Number of participants who responded to treatment after 8 weeks; measured as at least $50 \%$ reduction of the total score on an standardised depression scale, in the following order: Hamilton Depression Scale (HDRS; Hamilton 1960), Montgomery-Åsberg Depression Rating Scale (MADRS; Montgomery 1979), Clinical Global Impression Improvement (CGI-I; Guy 1970), or any other validated depression scale (Furukawa 2016). The response rate will be calculated based on the total number of randomised participants.

2. Number of dropouts due to any cause after 8 weeks.

3. Number of dropouts due to any side effect after 8 weeks.

\section{Secondary outcomes}

1. Number of participants who responded to treatment after 2 weeks and after 18 weeks.

2. Number of participants who achieved remission after 8 weeks, measured by these depression scales (in the following order, depending on availability):

a. 7 or fewer points on the HDRS-17 (or 8 or fewer on any other HDRS-scale);

b. 12 or fewer points on the MADRS;

c. A score of 'not ill' or 'borderline mentally ill' (score 1 or 2) on the CGI-Severity.

3. Change in depressive symptoms between baseline and 8 weeks

4. Safety:

a. total number of participants with any adverse events;

b. total number of participants experiencing any of the following side effects: akathisia, anxiety, decreased blood cortisol, cardiovascular events (such as stroke or myocardial infarction), constipation, dizziness, fatigue, headache, increased appetite, weight gain, nasopharyngitis (inflammation of the nose and throat), leukopenia (reduction of the number of white blood cells below reference range), neutropenia (reduction of the number of neutrophils below reference range), agranulocytosis (a more severe reduction of granulocytes below reference range), metabolic changes (such as hyperglycaemia, diabetes mellitus, dyslipidaemia), mortality, suicidal thoughts and behaviour, neuroleptic malignant syndrome (NMS; a rare, life-threatening reaction that can occur in response to neuroleptic or antipsychotic medication), pathological gambling and other compulsive behaviours, restlessness, somnolence, tardive dyskinesia (stiff, jerky, uncontrollable movements of one's face and body), tremor. In order to avoid missing any uncommon but important side effects, we will report any other side effects extracted from the included studies.

Side effects will be considered from the beginning of the intervention until the end of the longest follow-up period of the included trials.

1. Social functioning or adjustment, evaluated by Sheehan Disability Scale (SDS: Sheehan 1983)

2. Health-related quality of life, evaluated by Quality of Life Enjoyment and Satisfaction Questionnaire-short form (Q-LESSF) (Stevanovic 2011)

These are the range of weeks considered for each timepoint:

- 2 weeks: we will give preference to 2 -week data, but we will consider the outcome between weeks 1 and 4 ( $\geq 1$ and $<4$ weeks) if 2 week data are not available;

- 8 weeks: we will give preference to 8 -week data, but we will consider the outcome between weeks 4 and 12 ( $\geq 4$ and $\leq 12$ weeks) if 8 week data are not available;

- 18 weeks: we will give preference to 18 -week data, but we will consider the outcome between weeks 12 and 24 ( $\geq 12$ and $\leq 24$ weeks) if 18 week data are not available.

\section{Search methods for identification of studies}

\section{Electronic searches}

We will search the following databases, using relevant subject headings (controlled vocabularies) and search syntax, appropriate to each resource:

- Cochrane Common Mental Disorders Controlled Trials Register (CCMDCTR; all available years);

- Cochrane Central Register of Controlled Trials (CENTRAL; current issue) in the Cochrane Library;

- Ovid MEDLINE (2000 onwards);

- Ovid Embase (2000 onwards);

- Ovid PsyciNFO (2000 onwards).

We will search the available resources from 2000 onwards as brexpiprazole's synthesis was announced in 2006 (Kumar 2018), and clinical trials before 2000 are highly unlikely. We will search the US National Institutes of Health $(\mathrm{NIH})$ database of clinical studies ClinicalTrials.gov (clinicaltrials.gov), the World Health Organization (WHO) International Clinical Trials Registry Platform (ICTRP) (apps.who.int/trialsearch/) and Lundbeck's clinical trials database (www.lundbeck.com/trials) for ongoing or unpublished trials.

We will search MEDLINE Ovid for human studies using the following terms: ((Brexpiprazole or OPC-34712 or Rexulti).mp. not (exp animals/ not humans.sh.)).

We will not apply a study design filter, so that the search will retrieve randomised controlled trials or adverse effects information, relevant to this review. 
We will place no restrictions on language or publication status to the searches.

\section{Searching other resources}

\section{Regulatory Data}

We will search regulatory data from the European Medicines Agency (EMA) and the FDA, the Medicines and Healthcare products Regulatory Agency in the UK, the Medicines Evaluation Board in the Netherlands, the Medical Products Agency in Sweden, the Pharmaceuticals and Medical Devices Agency (PMDA) in Japan, the Therapeutic Goods Administration (TGA) in Australia, and The National Medical Products Administration (NMPA) in China.

\section{Reference lists}

We will check the reference lists of all included trials and relevant systematic reviews to identify additional trials missed from the electronic searches (for example, unpublished or in-press citations).

\section{Correspondence}

We will contact trial authors and subject experts for information on unpublished or ongoing trials, or to request additional trial data.

\section{Data collection and analysis}

\section{Selection of studies}

Two review authors (SR, FDC, or IK) will independently screen titles and abstracts to include all potential studies we identify as a result of the search; we will code them as 'retrieve' (eligible or potentially eligible or unclear), or 'do not retrieve'. If there are any disagreements, a fourth author will be asked to arbitrate (AC). We will retrieve the full-text study reports or publications, and two review authors (SR, FDC, or IK) will independently screen the fulltext report, identify studies for inclusion, and identify and record reasons for exclusion of the ineligible studies. We will resolve any disagreement through discussion, or if required, we will consult a fourth author (AC). We will identify and exclude duplicates, and collate multiple reports of the same study, so that each study, rather than each report, is the unit of interest in the review. We will record the selection process in sufficient detail to complete a PRISMA flow diagram, and table describing the characteristics of excluded studies (Liberati 2009).

\section{Data extraction and management}

Two review authors (SR, FDC, or IK) will independently extract study characteristics from included studies. We will extract the following study characteristics:

1. Methods: study design, total duration of study, number of study centres and location, study setting, and date of study

2. Participants: $\mathrm{N}$ randomised, $\mathrm{N}$ lost to follow-up or withdrawn, $\mathrm{N}$ analysed, mean age, age range, gender, severity of condition, diagnostic criteria, inclusion criteria, and exclusion criteria

3. Interventions: intervention, comparison, concomitant medications, and excluded medications

4. Outcomes: outcomes specified and collected, and time points reported

5. Notes: funding for trial, and notable conflicts of interest of trial authors
Two review authors (SR, IK) will independently extract outcome data from included studies. We will resolve disagreements by consensus, or by involving a third person (AC). One review author (SR) will transfer data into the Review Manager 5 (Review Manager 2020) file. We will double-check that data are entered correctly by comparing the data presented in the systematic review with the data extraction form.

\section{Assessment of risk of bias in included studies}

Two review authors (SR, FDC, or IK) will independently assess risk of bias for each study, using version one of the Cochrane 'Risk of bias' tool (RoB), outlined in the Cochrane Handbook for Systematic Reviews of Interventions (Higgins 2011). We will resolve any disagreements by discussion, or by involving another author $(A C)$. We will assess the risk of bias of the specific results of a trial according to the following domains:

1. Selection bias: random sequence generation; allocation concealment

2. Performance bias: blinding of participants and personnel

3. Detection bias: blinding of outcome assessment

4. Attrition bias: incomplete outcome data (attrition rate $\geq 30 \%$ will be considered high risk of bias)

5. Reporting bias: selective reporting, and

6. Other bias: other sources of bias

When considering treatment effects, we will take into account the risk of bias for the studies that contribute to that outcome.

\section{Measures of treatment effect}

We will analyse dichotomous data as odds ratios (OR), with $95 \%$ confidence intervals, and continuous data as mean difference (MD) or standardised mean difference (SMD) with 95\% confidence intervals. We will calculate SMDs when different scales are used to measure the same outcome (e.g. HDRS and MADRS).

We will narratively describe skewed data reported as medians and interquartile ranges.

\section{Unit of analysis issues}

We consider participants as the unit of analysis. For cross-over studies, we will extract data from the first period only, as carry-over effects can be important in antidepressant trials (Spineli 2013).

\section{Dealing with missing data}

We will contact investigators or study sponsors in order to verify key study characteristics, and obtain missing numerical outcome data where possible (e.g. when a study is identified as abstract only). Where possible, we will use the Revman 5 calculator to calculate missing standard deviations using other data from the trial, such as confidence intervals, based on methods outlined in the Handbook (Higgins 2020a). When this is not possible, and the missing data are thought to introduce serious bias, we will explore the impact of including such studies in the overall assessment of results by a sensitivity analysis.

\section{Assessment of heterogeneity}

We will inspect forest plots visually to consider the direction and magnitude of effects and the degree of overlap between confidence intervals. We will use the $I^{2}$ statistic to quantify inconsistency 
among the trials in each analysis. We will also consider the $P$ value from the $\mathrm{Chi}^{2}$ test. If we identify substantial heterogeneity, we will report it and explore possible causes by prespecified subgroup analysis. We will use the following thresholds for the interpretation of the statistics (Deeks 2019):

- $0 \%$ to $40 \%$ : might not be significant;

- $30 \%$ to $60 \%$ : may represent moderate heterogeneity;

- $50 \%$ to $90 \%$ : may represent substantial heterogeneity;

- $75 \%$ to $100 \%$ : considerable heterogeneity.

The size and importance of the $\mathrm{I}^{2}$ statistic will be interpreted in the context of sample size, the magnitude and the direction of the treatment effects, and the strength of evidence (Higgins 2020b).

\section{Assessment of reporting biases}

If we are able to pool more than 10 trials, we will create and examine a funnel plot to explore possible small study biases for the primary outcomes, and will perform a formal statistical test for asymmetry.

\section{Data synthesis}

We will analyse data using Review Manager 5.4 software (Review Manager 2020). We will undertake meta-analyses only where this is meaningful, i.e. if the treatments, participants, and the underlying clinical question are similar enough for pooling to make sense.

We will use a random-effects model for dichotomous and continuous outcomes, which has been empirically proven to have a higher generalisability than other summary of effects measures (Furukawa 2002).

\section{Subgroup analysis and investigation of heterogeneity}

We plan to carry out the following subgroup analyses using age and dosage as variables of interest because young people (children and adolescents) can respond differently to antidepressants than individuals aged 18 or older (Cipriani 2016) and flexible dosage regimens versus fixed dosage regimens of antidepressants might differ in terms of efficacy (Furukawa 2020).

- Age groups:

* participants below 18 years of age

* participants 18 years old or older

- Dosage groups:

* Fixed dosage - participants are assigned to a specific dose of a treatment; dosage adjustment is not allowed

* Flexible dosage - dosage adjustments are allowed

\section{Sensitivity analysis}

We plan to carry out the following sensitivity analyses:

- excluding trials in which allocation concealment is not clearly described, or double-blinding is unclear (Furukawa 2016)

- excluding trials with dropout rates of more than $20 \%$ in one of the included treatment arms (Furukawa 2016)

- excluding trials with imputed data (Furukawa 2005), and those for which the standard deviation was missing and we imputed it from other trials (Furukawa 2006)

- excluding trials with one or more domains with high risk of bias

\section{Reaching conclusions}

We will base our conclusions only on the findings from the quantitative or narrative synthesis of studies included in this review. We will avoid making recommendations for practice; our implications for research will suggest priorities for future research and outline the remaining uncertainties in the area.

\section{Summary of findings and assessment of the certainty of the evidence}

We will create a 'Summary of findings' table using the following outcomes, including the two most frequent adverse events.

- Number of participants who responded to treatment at eight weeks

- Number of dropouts due to any cause at eight weeks

- Number of dropouts due to any side effect at eight weeks

- Remission rates at eight weeks

- Change in depressive symptoms between baseline and eight weeks

- Side effect: akathisia

- Side effect: weight gain

We will use the methods and recommendations described in Chapter 14 of the Cochrane Handbook for Systematic Reviews of Interventions (Schünemann 2019), and GRADEpro GDT software (GRADEpro GDT). We will justify all decisions to downgrade the quality of the evidence using footnotes and we will make comments to aid reader's understanding of the review where necessary.

Factors that can reduce the quality of evidence:

- Limitations in study design or execution (risk of bias): reduction by 1 or 2 levels

- Inconcistency of results: reduction by 1 or 2 levels

- Indirectness of evidence: reduction by 1 or 2 levels

- Imprecision: reduction by 1 or 2 levels

- Publication bias: reduction by 1 or 2 levels

Two review authors (SR, IK) will independently assess the quality of the evidence, with disagreements resolved by discussion or involving a third author (AC). They will justify their judgements will be justified, document them, and we will incorporated them into reporting of results for each outcome.

\section{ACKNOWLEDGEMENTS}

We would like to acknowledge the support of the editorial team of the Cochrane Common Mental Disorders Group (CCMD) in reviewing and commenting on the draft protocol, and in particular Sarah Dawson, the Information Specialist for the Group, who helped develop the search strategies.

The authors and the CCMD Editorial Team are grateful to the following peer reviewers for their time and comments: Simon Davies, Lindsay Robertson, Jean Sellar-Edmunds, and Ellen Van Leeuwen. They would also like to thank Cochrane Copy Edit Support for the team's help.

CCMD funding acknowledgement: the National Institute for Health Research (NIHR) is the largest single funder of the CCMD Group. 
Disclaimer: the views and opinions expressed herein are those of the review authors, and do not necessarily reflect those of the NIHR, the NHS, or the Department of Health and Social Care. 


\section{R E F E E N CE S}

\section{Additional references}

\section{APA 1994}

American Psychiatric Association. Diagnostic and Statistical Manual of Mental Disorders (DSM-IV). 4th edition. Washington (DC): American Psychiatric Association, 1994.

\section{APA 2000}

American Psychiatric Association. Diagnostic and Statistical Manual of Mental Disorders (DSM-IV-TR). 4th text revised edition. Washington (DC): American Psychiatric Association, 2000.

\section{APA 2010}

Gelenberg AJ, Freeman MP, Markowitz JC, Rosenbaum JF, Thase ME, Trivedi MJ, et al, Work Group on Major Depressive Disorder. American Psychiatric Association practice guideline for the treatment of major depressive disorder. 3rd Edition. psychiatryonline.org/pb/assets/raw/sitewide/ practice_guidelines/guidelines/mdd.pdf 2010.

\section{APA 2013}

American Psychiatric Association. Diagnostic and Statistical Manual of Mental Disorders: DSM-5. 5th edition. Washington (DC): American Psychiatric Association, 2013.

\section{Bauer 2002}

Bauer M, Whybrow PC, Angst J, Versiani M, Moller HJ, World Federation of Societies Biological Psychiatry Task Force on Treatment Guidelines for Unipolar Depressive Disorders. World Federation of Societies of Biological Psychiatry (WFSBP) guidelines for biological treatment of unipolar depressive disorders. Part 1. Acute and continuation treatment of major depressive disorder. World Journal of Biological Psychiatry 2002;3(1):5-43.

\section{Cipriani 2016}

Cipriani A, Zhou X, Del Giovane C, Hetrick SE, Qin B, Whittington $C$, et al. Comparative efficacy and tolerability of antidepressants for major depressive disorder in children and adolescents: a network meta-analysis. Lancet 2016 Aug;388(10047):881-90.

\section{Cipriani 2018}

Cipriani A, Furukawa TA, Salanti G, Chaimani A, Atkinson LZ, Ogawa Y, et al. Comparative efficacy and acceptability of 21 antidepressant drugs for the acute treatment of adults with major depressive disorder: a systematic review and network meta-analysis. Lancet 2018;391(10128):1357-66.

\section{Citrome 2015}

Citrome L. Brexpiprazole for schizophrenia and as adjunct for major depressive disorder: a systematic review of the efficacy and safety profile for this newly approved antipsychotic - what is the number needed to treat, number needed to harm and likelihood to be helped or harmed? International Journal of Clinical Practice 2015;69(9):978-97.

\section{Deeks 2019}

Deeks JJ, Higgins JPT, Altman DG, editor(s). Chapter 10: Analysing data and undertaking meta-analyses. In: Higgins JPT, Thomas J, Chandler J, Cumpston M, Li T, Page MJ, et al, editor(s). Cochrane Handbook for Systematic Reviews of Interventions version 6.0 (updated July 2019). Cochrane. 2019. Available from training.cochrane.org/handbook.

\section{EMA 2018}

European Medicines Agency. Rxulti (brexpiprazole): summary of opinion (initial authorisation). www.ema.europa.eu/en/ documents/smop-initial/chmp-summary-positive-opinionrxulti_en.pdf; ((accessed 8 June 2020)).

\section{Fava 2017}

Fava M, Okame T, Matsushima Y, Perry P, Weiller E, Baker RA. Switching from inadequate adjunctive or combination treatment options to brexpiprazole adjunctive to antidepressant: an open-label study on the effects on depressive symptoms and cognitive and physical functioning. International Journal of Neuropsychopharmacology 2017;20(1):22-30.

\section{Fava 2020}

Fava GA, Cosci F, Guidi J, Rafanelli C. The deceptive manifestations of treatment resistance in depression: A new look at the problem. Psychotherapy and Psychosomatics 2020;89(5):265-73.

\section{FDA 2007}

US Food and Drug Administration. Abilify (aripiprazole): NDA application approval. www.accessdata.fda.gov/ drugsatfda_docs/appletter/2007/021436s018,\%20021866s005, \%20021729s005,\%20021713s013ltr.pdf (accessed 4 April 2020).

\section{FDA 2015}

US Food and Drug Administration. Rexulti (brexpiprazole): NDA application approval. www.accessdata.fda.gov/ drugsatfda_docs/appletter/2015/205422Orig1Orig2s000ltr.pdf (accessed 4 April 2020).

\section{Feighner 1972}

Feighner JP, Robins E, Guze SB, Woodruff RA, Winokur G, Munoz R. Diagnostic criteria for use in psychiatric research. Archives of General Psychiatry 1972;26(1):57-63.

\section{Furukawa 2002}

Furukawa TA, Guyatt GH, Griffith LE. Can we individualize the 'number needed to treat'? An empirical study of summary effect measures in meta-analyses. International Journal of Epidemiology 2002;31(1):72-6.

\section{Furukawa 2005}

Furukawa TA, Cipriani A, Barbui C, Brambilla P, Watanabe N. Imputing response rates from means and standard deviations in meta-analysis. International Clinical Psychopharmacology 2005;20(1):49-52. 


\section{Furukawa 2006}

Furukawa TA, Barbui C, Cipriani A, Brambilla P, Watanabe N. Imputing missing standard deviations in meta-analyses can provide accurate results. Journal of Clinical Epidemiology 2006;59(1):7-10.

\section{Furukawa 2016}

Furukawa TA, Salanti G, Atkinson LZ, Leucht S, Ruhe HG, Turner EH. Comparative efficacy and acceptability of firstgeneration and second-generation antidepressants in the acute treatment of major depression: protocol for a network metaanalysis. BMJ Open 2016;6(7):e010919.

\section{Furukawa 2020}

Furukawa TA, Salanti G, Cowen PJ, Leucht S, Cipriani A. No benefit from flexible titration above minimum licensed dose in prescribing antidepressants for major depression: systematic review. Acta Psychiatrica Scandinavica 2020;141(5):401-9. [DOI: 10.1111/acps.13145]

\section{GBD 2017}

GBD 2017 Disease and Injury Incidence and Prevalence Collaborators. Global, regional, and national incidence, prevalence, and years lived with disability for 354 diseases and injuries for 195 countries and territories, 1990-2017: a systematic analysis for the Global Burden of Disease Study 2017. Lancet 2017;392(10159):1789-858.

\section{GRADEpro GDT [Computer program]}

McMaster University (developed by Evidence Prime) GRADEpro GDT. Version accessed prior to 7 December 2020. Hamilton (ON): McMaster University (developed by Evidence Prime). Available at gradepro.org.

\section{Guy 1970}

Guy W, Bonato RR. Manual for the ECDEU Assessment Battery. 2 edition. Chevy Chase (Md): U.S. Department of Health, Education, and Welfare, National Institute of Mental Health, 1970.

\section{Hamilton 1960}

Hamilton M. A rating scale for depression. Journal of Neurology, Neurosurgery and Psychiatry 1960;23:56-62.

\section{Higgins 2011}

Higgins JPT, Altman DG, Sterne JAC, editor(s). Chapter 8: Assessing risk of bias in included studies. In: Higgins JPT, Green S, editor(s). Cochrane Handbook for Systematic Reviews of Interventions Version 5.1.0 (updated March 2011). The Cochrane Collaboration, 2011. Available from training.cochrane.org/ handbook/archive/v5.1.

\section{Higgins 2020a}

Higgins JPT, Li T, Deeks JJ. Chapter 6: Choosing effect measures and computing estimates of effect. In: Higgins JPT, Thomas J, Chandler J, Cumpston M, Li T, Page MJ, Welch VA, editor(s). Cochrane Handbook for Systematic Reviews of Interventions version 6.1 (updated September 2020). Cochrane, 2020. Available from training.cochrane.org/handbook.

\section{Higgins 2020b}

Deeks JJ, Higgins JPT, Altman DG. Chapter 10: Analysing data and undertaking meta-analyses. In: Higgins JPT, Thomas J, Chandler J, Cumpston M, Li T, Page MJ, Welch VA, editor(s). Cochrane Handbook for Systematic Reviews of Interventions version 6.1 (updated September 2020). Cochrane, 2020. Available from training.cochrane.org/handbook.

\section{Kennedy 2016}

Kennedy SH, Lam RW, McIntyre RS, Tourjman SV, Bhat V, Blier P, et al. Canadian network for mood and anxiety treatments (CANMAT) 2016 clinical guidelines for the management of adults with major depressive disorder: section 3. Pharmacological treatments. Canadian Journal of Psychiatry 2016;61(9):540-60.

\section{Kishi 2019}

Kishi T, Sakuma K, Nomura I, Matsuda Y, Mishima K, Iwata N. Brexpiprazole as adjunctive treatment for major depressive disorder following treatment failure with at least one antidepressant in the current episode: a systematic review and meta-analysis. International Journal of Neuropsychopharmacology 2019;22(11):698-709.

\section{Kumar 2018}

Kumar AS, Kandanur SGS, Sen S, Oruganti S. Delineating an alternate convergent synthesis of brexpiprazole: a novel use of commercial 6,7-dihydrobenzo[b]thiophen-4(5H)-one as precursor to an efficacious Buchwald-Hartwig amination step. Journal of Chemical Sciences 2018;130:72.

\section{Liberati 2009}

Liberati A, Altman DG, Tetzlaff J, Mulrow C, Gotzsche PC, loannidis JP, et al. The PRISMA statement for reporting systematic reviews and meta-analyses of studies that evaluate health care interventions: explanation and elaboration. PLOS Medicine 2009;6(7):e1000100.

\section{Maeda 2014a}

Maeda K, Sugino H, Akazawa H, Amada N, Shimada J, Futamura T, et al. Brexpiprazole I: in vitro and in vivo characterization of a novel serotonin-dopamine activity modulator. Journal of Pharmacology and Experimental Therapeutics 2014;350(3):589-604.

\section{Maeda 2014b}

Maeda K, Lerdrup L, Sugino H, Akazawa H, Amada N, McQuade RD, et al. Brexpiprazole II: antipsychotic-like and procognitive effects of a novel serotonin-dopamine activity modulator. Journal of Pharmacology and Experimental Therapeutics 2014;350(3):605-14.

\section{Malhi 2016}

Malhi G S, Byrow Y. Is treatment-resistant depression a useful concept? Evidence-Based Mental Health 2016;19(1):1-3.

\section{McIntyre 2016}

McIntyre RS, Weiller E, Zhang P, Weiss C. Brexpiprazole as adjunctive treatment of major depressive disorder with anxious distress: results from a post-hoc analysis of two randomised controlled trials. Journal of Affective Disorders 2016;201:116-23. 


\section{Montgomery 1979}

Montgomery SA, Asberg M. A new depression scale designed to be sensitive to change. British Journal of Psychiatry 1979;134:382-9.

\section{NCT01360632}

NCT01360632. Study of the safety and efficacy of two fixed doses of OPC-34712 as adjunctive therapy in the treatment of adults with major depressive disorder (the Polaris Trial). clinicaltrials.gov/ct2/show/NCT01360632 (first posted 25 May 2011).

\section{NCT01360645}

NCT01360645. Study of the safety and efficacy of fixed dose OPC-34712 as adjunctive therapy in the treatment of adults with major depressive disorder (the Pyxis Trial). clinicaltrials.gov/ ct2/show/NCT01360645 (first posted 25 May 2011).

\section{Nelson 2018}

Nelson JC, Weiller E, Zhang P, Weiss C, Hobart M. Efficacy of adjunctive brexpiprazole on the core symptoms of major depressive disorder: a post hoc analysis of two pooled clinical studies. Journal of Affective Disorders 2018;227:103-8.

\section{NICE 2009}

National Institute for Health and Clinical Excellence. Depression in adults: recognition and management. Clinical guideline CG90. www.nice.org.uk/guidance/cg90 (accessed prior to 7 December 2020).

\section{Otsuka 2020}

Otsuka Pharmaceutical Co, Ltd. Highlights of prescribing information for Rexulti. www.otsuka-us.com/media/static/ Rexulti-PI.pdf;(accessed 4 April 2020).

\section{Papakostas 2020}

Papakostas GI, Jackson WC, Rafeyan R, Trivedi MH. Inadequate response to antidepressant treatment in major depressive disorder. Journal of Clinical Psychiatry 2020;81(3):OT19037COM5.

\section{Review Manager 2020 [Computer program]}

Nordic Cochrane Centre, The Cochrane Collaboration Review Manager 5 (RevMan 5). Version 5.4.1. Copenhagen: Nordic Cochrane Centre, The Cochrane Collaboration, 2020.

\section{Sadock 2015}

Sadock BJ, Sadock VA, Ruiz P. Kaplan \& Sadock's Synopsis of Psychiatry: Behavioral Sciences/Clinical Psychiatry. 11th edition. Philadelphia (Pa): Lippincott Williams and Wilkins, 2015.

\section{Salanti 2016}

Salanti G, Chaimani A, Furukawa TA, Higgins JPT, Ogawa Y, Cipriani $A$, et al. Impact of placebo arms on outcomes in antidepressant trials: systematic review and meta-regression analysis. International Journal of Epidemiology 2018;47(5):145464.

\section{Schünemann 2019}

Schünemann HJ, Higgins JP, Vist GE, Glasziou P, Akl EA, Skoetz N, et al. Chapter 14: Completing 'Summary of findings' tables and grading the certainty of the evidence. In: Higgins JP, Thomas J, Chandler J, Cumpston MS, Li T, Page MJ, et al, editor(s), Cochrane Handbook for Systematic Reviews of Interventions version 6.0 (updated October 2019). Cochrane, 2019. Available from www.training.cochrane.org/handbook.

\section{Sheehan 1983}

Sheehan DV. The Anxiety Disease. New York: Charles Scribner and Sons, 1983.

\section{Spineli 2013}

Spineli LM, Higgins JP, Cipriani A, Leucht S, Salanti G. Evaluating the impact of imputations for missing participant outcome data in a network meta-analysis. Clinical Trials 2013;10(3):378-88.

\section{Spitzer 1978}

Spitzer RL, Endicott J, Robins E. Research diagnostic criteria: rationale and reliability. Archives of General Psychiatry 1978;35(6):773-82.

\section{Stahl 2016}

Stahl SM. Mechanism of action of brexpiprazole: comparison with aripiprazole. CNS Spectrums 2016;21(1):1-6.

\section{Stevanovic 2011}

Stevanovic D. Quality of Life Enjoyment and Satisfaction Questionnaire-short form for quality of life assessments in clinical practice: A psychometric study. Journal of Psychiatric and Mental Health Nursing 2011;18(8):744-50.

\section{Thase 2016}

Thase ME, Zhang P, Skuban A, Hobart M, Weiss C, Weiller E, et al. Efficacy of adjunctive brexpiprazole in patients with major depressive disorder: a clinical overview. Current Psychiatry Reviews 2016;12:291-301.

\section{Thase 2019}

Thase ME, Zhang P, Weiss C, Meehan SR, Hobart M. Efficacy and safety of brexpiprazole as adjunctive treatment in major depressive disorder: overview of four short-term studies. Expert Opinion on Pharmacotherapy 2019;20(15):1907-16.

\section{WHO 1978}

World Health Organization. The Ninth Revision of the International Classification of Diseases and Related Health Problems (ICD-9). Geneva: World Health Organisation, 1978.

\section{WHO 1992}

World Health Organization. The Tenth Revision of the International Classification of Diseases and Related Health Problems (ICD-10). Geneva: World Health Organisation, 1992.

\section{Yoon 2017}

Yoon S, Jeon SW, Ko YH. Adjunctive brexpiprazole as a novel effective strategy for treating major depressive disorder: a systematic review and meta-analysis. Journal of Clinical Psychopharmacology 2017;37(1):46-53. 


\section{HISTORY}

Protocol first published: Issue 2, 2021

\section{CONTRIBUTIONS OFAUTHORS}

SR: overall responsibility for the protocol, protocol writing and methodological design.

IK: supervised the draft, and critically reviewed the protocol.

FDC: critically reviewed the protocol.

PM: critically reviewed the protocol.

AC: protocol writing, and overall supervision of the review process.

\section{DECLARATIONS OF INTEREST}

SR: none known

\section{IK: none known}

FDC: none known

PM: has received payment from Lunbeck Export A/S for educational presentations on schizophrenia.

AC: has received research and consultancy fees from INCiPiT (Italian Network for Paediatric Trials), CARIPLO Foundation and Angelini Pharma.

\section{SOURCES OF SUPPORT}

\section{Internal sources}

- University of Oxford, UK

\section{External sources}

- National Institute for Health Research (NIHR), UK

Andrea Cipriani is supported by the NIHR Oxford Cognitive Health Clinical Research Facility, by an NIHR Research Professorship (grant RP-2017-08-ST2-006), by the NIHR Oxford and Thames Valley Applied Research Collaboration, and by the NIHR Oxford Health Biomedical Research Centre (grant BRC-1215-20005). 